\title{
Some new oscillation criteria of fourth-order quasi-linear differential equations with neutral term
}

\author{
Saeed Althubiti ${ }^{1}$, Fahad Alsharari ${ }^{2,3}$, Omar Bazighifan ${ }^{4 *}$ (D) and George E. Chatzarakis ${ }^{5}$
}

\section{"Correspondence:}

o.bazighifan@gmail.com

${ }^{4}$ Department of Mathematics,

Faculty of Science, Hadhramout

University, Hadhramout, 50512, Yemen

Full list of author information is available at the end of the article

\begin{abstract}
In this article, we are interested in studying the asymptotic behavior of fourth-order neutral differential equations. Despite the growing interest in studying the oscillatory behavior of delay differential equations of second-order, fourth-order equations have received less attention. We get more than one criterion to check the oscillation by the generalized Riccati method and the integral average technique. Our results are an extension and complement to some results published in the literature. Examples are given to prove the significance of new theorems.
\end{abstract}

Keywords: Fourth-order differential equations; Neutral delay; Oscillation; Philos-type oscillation

\section{Introduction}

In this paper, we investigate the oscillation properties of solutions to the fourth-order neutral differential equations:

$$
\left(z(x) \varsigma_{r_{1}}\left(\delta^{\prime \prime \prime}(x)\right)\right)^{\prime}+\tilde{\omega}(x) \varsigma_{r_{2}}(\beta(\theta(x)))=0,
$$

where $\varsigma_{r_{i}}[s]=|s|^{r_{i}-1} s, \delta(x)=\beta(x)+\tilde{y}(x) \beta(\tilde{\theta}(x))$. Throughout this paper, we suppose that:

$\left(S_{1}\right) r_{1}$ and $r_{2}$ are quotients of odd positive integers,

$\left(S_{2}\right) z, \tilde{y}, \tilde{\omega} \in C\left[x_{0}, \infty\right), z(x)>0, z^{\prime}(x) \geq 0, \tilde{\omega}(x)>0,0 \leq \tilde{y}(x) \leq \tilde{y}_{0}<1, \tilde{\theta}, \theta \in C\left[x_{0}, \infty\right)$, $\tilde{\theta}(x) \leq x, \lim _{x \rightarrow \infty} \tilde{\theta}(x)=\lim _{x \rightarrow \infty} \theta(x)=\infty$,

and under the assumption

$$
\int_{x_{0}}^{\infty} \frac{1}{z^{1 / r_{1}}(s)} \mathrm{d} s=\infty
$$

Definition $1.1([1])$ Let

$$
D=\left\{(x, s) \in \mathbb{R}^{2}: x \geq s \geq x_{0}\right\} \text { and } D_{0}=\left\{(x, s) \in \mathbb{R}^{2}: x>s \geq x_{0}\right\} .
$$

The function $G_{i} \in C(D, \mathbb{R})$ fulfills the following conditions:

(c) The Author(s) 2021. This article is licensed under a Creative Commons Attribution 4.0 International License, which permits use, sharing, adaptation, distribution and reproduction in any medium or format, as long as you give appropriate credit to the original author(s) and the source, provide a link to the Creative Commons licence, and indicate if changes were made. The images or other third party material in this article are included in the article's Creative Commons licence, unless indicated otherwise in a credit line to the material. If material is not included in the article's Creative Commons licence and your intended use is not permitted by statutory regulation or exceeds the permitted use, you will need to obtain permission directly from the copyright holder. To view a copy of this licence, visit http://creativecommons.org/licenses/by/4.0/. 
(i) $G_{i}(x, s)=0$ for $x \geq x_{0}, G_{i}(x, s)>0,(x, s) \in D_{0}$;

(ii) The functions $h, v \in C^{1}\left(\left[x_{0}, \infty\right),(0, \infty)\right)$ and $g_{i} \in C\left(D_{0}, \mathbb{R}\right)$ such that

$$
\frac{\partial}{\partial s} G_{1}(x, s)+\frac{\alpha^{\prime}(s)}{\alpha(s)} G(x, s)=g_{1}(x, s) G_{1}^{r_{1} /\left(r_{1}+1\right)}(x, s)
$$

and

$$
\frac{\partial}{\partial s} G_{2}(x, s)+\frac{h^{\prime}(s)}{h(s)} G_{2}(x, s)=g_{2}(x, s) \sqrt{G_{2}(x, s)} .
$$

Theory of oscillation of differential equations is a fertile study area and has attracted the attention of many authors recently. This is due to the existence of many important applications of this theory in neural networks, biology, social sciences, engineering, etc., see [2-10]. Very recently, a great development was found in the study of oscillation of solutions to neutral differential equations, see [11-20]. In particular, quasilinear/EmdenFowler differential equations have numerous applications in physics and engineering (e.g., quasilinear/Emden-Fowler differential equations arise in the study of $p$-Laplace equations, porous medium problems, and so on); see, e.g., the papers [5, 21-24] for more details, the papers [5, 6, 25-28] for the oscillation of quasilinear/Emden-Fowler differential equations, and the papers $[4,24,29-35]$ for the oscillation and asymptotic behavior of quasilinear/Emden-Fowler differential equations with different neutral coefficients.

Xing et al. [33] presented criteria for oscillation of the equation

$$
\left(z(x)\left(\delta^{(n-1)}(x)\right)^{r_{1}}\right)^{\prime}+\tilde{\omega}(x) \beta^{r_{1}}(\theta(x))=0
$$

under the conditions

$$
\left(\theta^{-1}(x)\right)^{\prime} \geq \theta_{0}>0, \quad \tilde{\theta}^{\prime}(x) \geq \tilde{\theta}_{0}>0, \quad \tilde{\theta}^{-1}(\theta(x))<x
$$

and

$$
\liminf _{x \rightarrow \infty} \int_{\tilde{\theta}^{-1}(\theta(x))}^{x} \frac{\widehat{\tilde{\omega}}(s)}{z(s)}\left(s^{n-1}\right)^{r_{1}} \mathrm{~d} s>\left(\frac{1}{\theta_{0}}+\frac{\tilde{y}_{0}^{r_{1}}}{\theta_{0} \tilde{\theta}_{0}}\right)>\frac{((n-1) !)^{r_{1}}}{\mathrm{e}},
$$

where $0 \leq \tilde{y}(x)<\tilde{y}_{0}<\infty$ and $\widehat{\widetilde{\omega}}(x):=\min \left\{\tilde{\omega}\left(\theta^{-1}(x)\right), \tilde{\omega}\left(\theta^{-1}(\tilde{\theta}(x))\right)\right\}$.

Bazighifan et al. [18], Li and Rogovchenko [25], and Zhang et al. [26, 28] presented oscillation results for fourth-order equation

$$
\left(z(x)\left(\delta^{\prime \prime \prime}(x)\right)^{r_{1}}\right)^{\prime}+\tilde{\omega}(x) \beta^{r_{1}}(\theta(x))=0
$$

under the condition

$$
\int_{x_{0}}^{\infty} \frac{1}{z^{1 / r_{1}}(s)} \mathrm{d} s<\infty
$$

and they used the Riccati technique.

Zhang et al. [36] established oscillation criteria for the equation

$$
\left(z(x)\left(\delta^{(n-1)}(x)\right)^{r_{1}}\right)^{\prime}+\tilde{\omega}(x) f(\beta(\theta(x)))=0
$$


and under the condition

$$
\int_{x_{0}}^{\infty}\left(k \rho(x) E(x)-\frac{1}{4 \lambda}\left(\frac{\rho^{\prime}(x)}{\rho(x)}\right)^{2} \eta(x)\right) \mathrm{d} z=\infty .
$$

By using the Riccati transformation technique, Chatzarakis et al. [19] established asymptotic behavior for the neutral equation

$$
\left(z(x)\left(\delta^{\prime \prime \prime}(x)\right)^{r_{1}}\right)^{\prime}+\int_{a}^{b} \tilde{\omega}(x, s) f(\beta(\theta(x, s))) \mathrm{d} s=0
$$

In this work, a new oscillation condition is created for fourth-order differential equations with a canonical operator. We use the Riccati technique and the integral averaging technique to prove our results.

Here are the notations used for our study:

$$
\begin{aligned}
& E_{1}(x)=\alpha(x) \tilde{\omega}(x)\left(1-\tilde{y}_{0}\right)^{r_{2}} A_{1}^{r_{2}-r_{1}}\left(\frac{\theta(x)}{x}\right)^{3 r_{2}}, \\
& \Phi(x)=\left(1-\tilde{y}_{0}\right)^{r_{2} / r_{1}} h(x) A_{2}^{r_{2} / r_{1}-1}(x) \int_{x}^{\infty}\left(\frac{1}{z(u)} \int_{u}^{\infty} \tilde{\omega}(s) \frac{\theta^{r_{2}}(s)}{s^{r_{2}}} \mathrm{~d} s\right)^{1 / r_{1}} \mathrm{~d} u
\end{aligned}
$$

and

$$
\Theta(x)=r_{1} \mu_{1} \frac{x^{2}}{2 z^{1 / r_{1}}(x) \alpha^{1 / r_{1}}(x)} .
$$

\section{Oscillation criteria}

We next present the lemmas needed for the proof of the original results.

Lemma 2.1 ([37]) If $\beta^{(i)}(x)>0, i=0,1, \ldots, n$, and $\beta^{(n+1)}(x)<0$, then

$$
n ! \frac{\beta(x)}{x^{n}} \geq(n-1) ! \frac{\beta^{\prime}(x)}{x^{n-1}}
$$

Lemma 2.2 ([20]) Let $\beta \in C^{n}\left(\left[x_{0}, \infty\right),(0, \infty)\right)$. Assume that $\beta^{(n)}(x)$ is of a fixed sign and not identically zero on $\left[x_{0}, \infty\right)$ and that there exists $x_{1} \geq x_{0}$ such that $\beta^{(n-1)}(x) \beta^{(n)}(x) \leq 0$ for all $x \geq x_{1}$. If $\lim _{x \rightarrow \infty} \beta(x) \neq 0$, then for every $\mu \in(0,1)$ there exists $x_{\mu} \geq x_{1}$ such that

$$
\beta(x) \geq \frac{\mu}{(n-1) !} x^{n-1}\left|\beta^{(n-1)}(x)\right| \quad \text { for } x \geq x_{\mu} .
$$

Lemma 2.3 ([27]) Let $a \geq 0$. Then

$$
X \beta-Y \beta^{(a+1) / a} \leq a^{a}(a+1)^{-(a+1)} Y^{-a} X^{a+1},
$$

where $Y>0$ and $X$ are constants.

Lemma $2.4([38])$ 
Then

Case $\left(\mathbf{N}_{1}\right): \quad \delta^{(j)}(x)>0 \quad$ for $j=0,1,2,3$,

Case $\left(\mathbf{N}_{2}\right): \quad \delta^{(j)}(x)>0 \quad$ for $j=0,1,3$ and $\delta^{\prime \prime}(x)<0$,

for $x \geq x_{1}$, where $x_{1} \geq x_{0}$ is sufficiently large.

Lemma 2.5 Let (5) hold. Then

$$
\left(z(x)\left(\delta^{\prime \prime \prime}(x)\right)^{r_{1}}\right)^{\prime} \leq-G(x)\left(\delta^{\prime \prime \prime}(\theta(x))\right)^{r_{2}},
$$

where

$$
G(x)=\tilde{\omega}(x)\left(1-\tilde{y}_{0}\right)^{r_{2}}\left(\frac{\mu}{6} \theta^{3}(x)\right)^{r_{2}} .
$$

Proof Let (5) hold. From the definition of $\delta$, we get

$$
\begin{aligned}
\beta(x) & \geq \delta(x)-\tilde{y}_{0} \beta(\tilde{\theta}(x)) \\
& \geq \delta(x)-\tilde{y}_{0} \delta(\tilde{\theta}(x)) \\
& \geq\left(1-\tilde{y}_{0}\right) \delta(x),
\end{aligned}
$$

which with (1) gives

$$
\left(z(x)\left(\delta^{\prime \prime \prime}(x)\right)^{r_{1}}\right)^{\prime}+\tilde{\omega}(x)\left(1-\tilde{y}_{0}\right)^{r_{2}} \delta^{r_{2}}(\theta(x)) \leq 0 .
$$

Using Lemma 2.2, we see that

$$
\delta(x) \geq \frac{\mu}{6} x^{3} \delta^{\prime \prime \prime}(x) .
$$

Combining (7) and (8), we find

$$
\left(z(x)\left(\delta^{\prime \prime \prime}(x)\right)^{r_{1}}\right)^{\prime}+\tilde{\omega}(x)\left(1-\tilde{y}_{0}\right)^{r_{2}}\left(\frac{\mu}{6} \theta^{3}(x)\right)^{r_{2}}\left(\delta^{\prime \prime \prime}(\theta(x))\right)^{r_{2}} \leq 0 .
$$

Thus, (6) holds. This completes the proof.

Lemma 2.6 Let (5) hold. If $\delta$ satisfies $\left(\mathbf{N}_{1}\right)$, then

$$
B^{\prime}(x) \leq \frac{\alpha^{\prime}(x)}{\alpha(x)} B(x)-E_{1}(x)-r_{1} \mu_{1} \frac{x^{2}}{2 z^{1 / r_{1}}(x) \alpha^{1 / r_{1}}(x)} B^{\frac{r_{1}+1}{r_{1}}}(x),
$$

if $\delta$ satisfies $\left(\mathbf{N}_{2}\right)$, then

$$
A^{\prime}(x) \leq-\Phi(x)+\frac{h^{\prime}(x)}{h(x)} A(x)-\frac{1}{h(x)} A^{2}(x)
$$


where

$$
B(x):=\alpha(x) \frac{z(x)\left(\delta^{\prime \prime \prime}(x)\right)^{r_{1}}}{\delta^{r_{1}}(x)}>0
$$

and

$$
A(x):=h(x) \frac{\delta^{\prime}(x)}{\delta(x)}, \quad x \geq x_{1}
$$

Proof Let (5) and ( $\left.\mathbf{N}_{1}\right)$ hold. From (11) and (7), we find

$$
B^{\prime}(x) \leq \frac{\alpha^{\prime}(x)}{\alpha(x)} B(x)-\alpha(x) \tilde{\omega}(x)\left(1-\tilde{y}_{0}\right)^{r_{2}} \frac{\delta^{r_{2}}(\theta(x))}{\delta^{r_{1}}(x)}-r_{1} \alpha(x) \frac{z(x)\left(\delta^{\prime \prime \prime}(x)\right)^{r_{1}}}{\delta^{r_{1}+1}(x)} \delta^{\prime}(x) .
$$

Using Lemma 2.1, we find

$$
\delta(x) \geq \frac{x}{3} \delta^{\prime}(x)
$$

and hence

$$
\frac{\delta(\theta(x))}{\delta(x)} \geq \frac{\theta^{3}(x)}{x^{3}}
$$

It follows from Lemma 2.2 that

$$
\delta^{\prime}(x) \geq \frac{\mu_{1}}{2} x^{2} \delta^{\prime \prime \prime}(x)
$$

for all $\mu_{1} \in(0,1)$ and every sufficiently large $x$. Thus, by (13), (14), and (15), we get

$$
\begin{aligned}
B^{\prime}(x) \leq & \frac{\alpha^{\prime}(x)}{\alpha(x)} B(x)-\alpha(x) \tilde{\omega}(x)\left(1-\tilde{y}_{0}\right)^{r_{2}} \delta^{r_{2}-r_{1}}(x)\left(\frac{\theta(x)}{x}\right)^{3 r_{2}} \\
& -r_{1} \mu_{1} \frac{x^{2}}{2 z^{1 / r_{1}}(x) \alpha^{1 / r_{1}}(x)} B^{\frac{r_{1}+1}{r_{1}}}(x) .
\end{aligned}
$$

Since $\delta^{\prime}(x)>0$, there exist $x_{2} \geq x_{1}$ and $A_{1}>0$ such that

$$
\delta(x)>A_{1} .
$$

Thus, we obtain

$$
\begin{aligned}
B^{\prime}(x) \leq & \frac{\alpha^{\prime}(x)}{\alpha(x)} B(x)-\alpha(x) \tilde{\omega}(x)\left(1-\tilde{y}_{0}\right)^{r_{2}} A^{r_{2}-r_{1}}\left(\frac{\theta(x)}{x}\right)^{3 r_{2}} \\
& -r_{1} \mu_{1} \frac{x^{2}}{2 z^{1 / r_{1}}(x) \alpha^{1 / r_{1}}(x)} B^{\frac{r_{1}+1}{r_{1}}}(x),
\end{aligned}
$$

which yields

$$
B^{\prime}(x) \leq \frac{\alpha^{\prime}(x)}{\alpha(x)} B(x)-E_{1}(x)-r_{1} \mu_{1} \frac{x^{2}}{2 z^{1 / r_{1}}(x) \alpha^{1 / r_{1}}(x)} B^{\frac{r_{1}+1}{r_{1}}}(x) .
$$

Thus, (9) holds. 
Let $\left(\mathbf{N}_{2}\right)$ hold. Integrating (7) from $x$ to $u$, we find

$$
z(u)\left(\delta^{\prime \prime \prime}(u)\right)^{r_{1}}-z(x)\left(\delta^{\prime \prime \prime}(x)\right)^{r_{1}} \leq-\int_{x}^{u} \tilde{\omega}(s)\left(1-\tilde{y}_{0}\right)^{r_{2}} \delta^{r_{2}}(\theta(s)) \mathrm{d} s .
$$

From Lemma 2.1, we obtain

$$
\delta(x) \geq x \delta^{\prime}(x)
$$

and hence

$$
\delta(\theta(x)) \geq \frac{\theta(x)}{x} \delta(x)
$$

For (17), letting $u \rightarrow \infty$ and using (18), we get

$$
z(x)\left(\delta^{\prime \prime \prime}(x)\right)^{r_{1}} \geq\left(1-\tilde{y}_{0}\right)^{r_{2}} \delta^{r_{2}}(x) \int_{x}^{\infty} \tilde{\omega}(s) \frac{\theta^{r_{2}}(s)}{s^{r_{2}}} \mathrm{~d} s .
$$

Integrating (19) from $x$ to $\infty$, we find

$$
\delta^{\prime \prime}(x) \leq-\left(1-\tilde{y}_{0}\right)^{r_{2} / r_{1}} \delta^{r_{2} / r_{1}}(x) \int_{x}^{\infty}\left(\frac{1}{z(u)} \int_{u}^{\infty} \tilde{\omega}(s) \frac{\theta^{r_{2}}(s)}{s^{r_{2}}} \mathrm{~d} s\right)^{1 / r_{1}} \mathrm{~d} u .
$$

From the definition of $A(x)$, we see that $A(x)>0$ for $x \geq x_{1}$, and using (16) and (20), we find

$$
\begin{aligned}
A^{\prime}(x)= & \frac{h^{\prime}(x)}{h(x)} A(x)+h(x) \frac{\delta^{\prime \prime}(x)}{\delta(x)}-h(x)\left(\frac{\delta^{\prime}(x)}{\delta(x)}\right)^{2} \\
\leq & \frac{h^{\prime}(x)}{h(x)} A(x)-\frac{1}{h(x)} A^{2}(x) \\
& -\left(1-\tilde{y}_{0}\right)^{r_{2} / r_{1}} h(x) \delta^{r_{2} / r_{1}-1}(x) \int_{x}^{\infty}\left(\frac{1}{z(u)} \int_{u}^{\infty} \tilde{\omega}(s) \frac{\theta^{r_{2}}(s)}{s^{r_{2}}} \mathrm{~d} s\right)^{1 / r_{1}} \mathrm{~d} u .
\end{aligned}
$$

Since $\delta^{\prime}(x)>0$, there exist $x_{2} \geq x_{1}$ and $A_{2}>0$ such that

$$
\delta(x)>A_{2}
$$

Thus, we obtain

$$
A^{\prime}(x) \leq-\Phi(x)+\frac{h^{\prime}(x)}{h(x)} A(x)-\frac{1}{h(x)} A^{2}(x)
$$

Thus, (10) holds. The proof of the theorem is completed.

Now, we present some Philos-type oscillation criteria for (1).

Theorem 2.7 Let (25) hold. If $\alpha, h \in C^{1}\left(\left[x_{0}, \infty\right), \mathbb{R}\right)$ such that

$$
\limsup _{x \rightarrow \infty} \frac{1}{G\left(x, x_{1}\right)} \int_{x_{1}}^{x} G(x, s) E_{1}(s)-\frac{g_{1}^{r_{1}+1}(x, s) G_{1}^{r_{1}}(x, s)}{\left(r_{1}+1\right)^{r_{1}+1}} \frac{2^{r_{1}} z(s) \alpha(s)}{\left(\mu_{1} s^{2}\right)^{r_{1}}} \mathrm{~d} s=\infty
$$


for all $\mu_{2} \in(0,1)$, and

$$
\limsup _{x \rightarrow \infty} \frac{1}{G_{2}\left(x, x_{1}\right)} \int_{x_{1}}^{x}\left(G_{2}(x, s) \Phi(s)-\frac{h(s) g_{2}^{2}(x, s)}{4}\right) \mathrm{d} s=\infty,
$$

then (1) is oscillatory.

Proof Let $\beta$ be a nonoscillatory solution of (1), we see that $\beta>0$. Assume that $\left(\mathbf{N}_{1}\right)$ holds. Multiplying (9) by $G(x, s)$ and integrating the resulting inequality from $x_{1}$ to $x$; we obtain

$$
\begin{aligned}
\int_{x_{1}}^{x} G(x, s) E_{1}(s) \mathrm{d} s \leq & B\left(x_{1}\right) G\left(x, x_{1}\right)+\int_{x_{1}}^{x}\left(\frac{\partial}{\partial s} G(x, s)+\frac{\alpha^{\prime}(s)}{\alpha(s)} G(x, s)\right) B(s) \mathrm{d} s \\
& -\int_{x_{1}}^{x} \Theta(s) G(x, s) B^{\frac{r_{1}+1}{r_{1}}}(s) \mathrm{d} s .
\end{aligned}
$$

From (3), we get

$$
\begin{aligned}
\int_{x_{1}}^{x} G(x, s) E_{1}(s) \mathrm{d} s \leq & B\left(x_{1}\right) G\left(x, x_{1}\right)+\int_{x_{1}}^{x} g_{1}(x, s) G_{1}^{r_{1} /\left(r_{1}+1\right)}(x, s) B(s) \mathrm{d} s \\
& -\int_{x_{1}}^{x} \Theta(s) G(x, s) B^{\frac{r_{1}+1}{r_{1}}}(s) \mathrm{d} s .
\end{aligned}
$$

Using Lemma 2.3 with $V=\Theta(s) G(x, s), U=g_{1}(x, s) G_{1}^{r_{1} /\left(r_{1}+1\right)}(x, s)$, and $\beta=B(s)$, we get

$$
\begin{gathered}
g_{1}(x, s) G_{1}^{r_{1} /\left(r_{1}+1\right)}(x, s) B(s)-\Theta(s) G(x, s) B^{\frac{r_{1}+1}{r_{1}}}(s) \\
\leq \frac{g_{1}^{r_{1}+1}(x, s) G_{1}^{r_{1}}(x, s)}{\left(r_{1}+1\right)^{r_{1}+1}} \frac{2^{r_{1}} z(x) \alpha(x)}{\left(\mu_{1} x^{2}\right)^{r_{1}}},
\end{gathered}
$$

which with (23) gives

$$
\frac{1}{G\left(x, x_{1}\right)} \int_{x_{1}}^{x}\left(G(x, s) E_{1}(s)-\frac{g_{1}^{r_{1}+1}(x, s) G_{1}^{r_{1}}(x, s)}{\left(r_{1}+1\right)^{r_{1}+1}} \frac{2^{r_{1}} z(s) \alpha(s)}{\left(\mu_{1} s^{2}\right)^{r_{1}}}\right) \mathrm{d} s \leq B\left(x_{1}\right)
$$

which contradicts (21).

Assume that $\left(\mathbf{N}_{2}\right)$ holds. Multiplying $(10)$ by $G_{2}(x, s)$ and integrating the resulting inequality from $x_{1}$ to $x$, we find

$$
\begin{aligned}
\int_{x_{1}}^{x} G_{2}(x, s) \Phi(s) \mathrm{d} s \leq & A\left(x_{1}\right) G_{2}\left(x, x_{1}\right) \\
& +\int_{x_{1}}^{x}\left(\frac{\partial}{\partial s} G_{2}(x, s)+\frac{h^{\prime}(s)}{h(s)} G_{2}(x, s)\right) A(s) \mathrm{d} s \\
& -\int_{x_{1}}^{x} \frac{1}{h(s)} G_{2}(x, s) A^{2}(s) \mathrm{d} s .
\end{aligned}
$$

Thus,

$$
\int_{x_{1}}^{x} G_{2}(x, s) \Phi(s) \mathrm{d} s \leq A\left(x_{1}\right) G_{2}\left(x, x_{1}\right)+\int_{x_{1}}^{x} g_{2}(x, s) \sqrt{G_{2}(x, s)} A(s) \mathrm{d} s
$$




$$
\begin{gathered}
-\int_{x_{1}}^{x} \frac{1}{h(s)} G_{2}(x, s) A^{2}(s) \mathrm{d} s \\
\leq A\left(x_{1}\right) G_{2}\left(x, x_{1}\right)+\int_{x_{1}}^{x} \frac{h(s) g_{2}^{2}(x, s)}{4} \mathrm{~d} s,
\end{gathered}
$$

and so

$$
\frac{1}{G_{2}\left(x, x_{1}\right)} \int_{x_{1}}^{x}\left(G_{2}(x, s) \Phi(s)-\frac{h(s) g_{2}^{2}(x, s)}{4}\right) \mathrm{d} s \leq A\left(x_{1}\right),
$$

which contradicts (22). The proof of the theorem completed.

Corollary 2.8 Let $(25)$ hold. If $\alpha, h \in C^{1}\left(\left[x_{0}, \infty\right)\right.$, $\left.\mathbb{R}\right)$ such that

$$
\int_{x_{0}}^{\infty}\left(E_{1}(s)-\frac{2^{r_{1}}}{\left(r_{1}+1\right)^{r_{1}+1}} \frac{z(s)\left(\alpha^{\prime}(s)\right)^{r_{1}+1}}{\mu_{1}^{r_{1}} s^{2 r_{1}} \alpha^{r_{1}}(s)}\right) \mathrm{d} s=\infty
$$

and

$$
\int_{x_{0}}^{\infty}\left(\Phi(s)-\frac{\left(h^{\prime}(s)\right)^{2}}{4 h(s)}\right) \mathrm{d} s=\infty
$$

for some $\mu_{1} \in(0,1)$ and every $A_{1}, A_{2}>0$, then (1) is oscillatory.

Example 2.9 Consider the equation

$$
\left(\beta+\frac{1}{2} \beta\left(\frac{1}{3} x\right)\right)^{(4)}+\frac{\tilde{\omega}_{0}}{x^{4}} \beta\left(\frac{1}{2} x\right)=0, \quad x \geq 1, \tilde{\omega}_{0}>0 .
$$

Let $r_{1}=r_{2}=1, z(x)=1, \tilde{y}(x)=1 / 2, \tilde{\theta}(x)=x / 3, \theta(x)=x / 2$, and $\tilde{\omega}(x)=\tilde{\omega}_{0} / x^{4}$. Hence, it is easy to see that

$$
\int_{x_{0}}^{\infty} \frac{1}{z^{1 / r_{1}}(s)} \mathrm{d} s=\infty, \quad E_{1}(x)=\frac{\tilde{\omega}_{0}}{16 s}
$$

and

$$
\Phi(x):=\frac{\tilde{\omega}_{0}}{24}
$$

If we put $\alpha(s):=x^{3}$ and $h(x):=x^{2}$, then we find

$$
\begin{gathered}
\int_{x_{0}}^{\infty}\left(E_{1}(s)-\frac{2^{r_{1}}}{\left(r_{1}+1\right)^{r_{1}+1}} \frac{z(s)\left(\alpha^{\prime}(s)\right)^{r_{1}+1}}{\mu_{1}^{r_{1}} s^{2 r_{1}} \alpha^{r_{1}}(s)}\right) \mathrm{d} s \\
=\int_{x_{0}}^{\infty}\left(\frac{\tilde{\omega}_{0}}{16 s}-\frac{9}{2 \mu_{1} s}\right) \mathrm{d} s
\end{gathered}
$$

and

$$
\int_{x_{0}}^{\infty}\left(\Phi(s)-\frac{\left(h^{\prime}(s)\right)^{2}}{4 h(s)}\right) \mathrm{d} s
$$




$$
=\int_{x_{0}}^{\infty}\left(\frac{\tilde{\omega}_{0}}{24}-1\right) \mathrm{d} s
$$

Thus,

$$
\tilde{\omega}_{0}>72
$$

and

$$
\tilde{\omega}_{0}>24 \text {. }
$$

From Corollary 2.8, equation (26) is oscillatory if $\tilde{\omega}_{0}>72$.

Example 2.10 Consider the equation

$$
\left(x\left(\beta+\tilde{y}_{0} \beta(\gamma x)\right)^{\prime \prime \prime}\right)^{\prime}+\frac{\tilde{\omega}_{0}}{x^{3}} \beta(\eta x)=0, \quad x \geq 1,
$$

where $\tilde{y}_{0} \in[0,1), \gamma, \eta \in(0,1)$, and $\tilde{\omega}_{0}>0$. Let $r_{1}=r_{2}=1, z(x)=x, \tilde{y}(x)=\tilde{y}_{0}, \tilde{\theta}(x)=\gamma x$, $\theta(x)=\eta x$, and $\tilde{\omega}(x)=\tilde{\omega}_{0} / x^{3}$. Hence, if we set $\alpha(s):=x^{2}$ and $h(x):=x$, then we get

$$
E_{1}(x)=\frac{\tilde{\omega}_{0}\left(1-\tilde{y}_{0}\right) \eta^{3}}{x}, \quad \Phi(x)=\frac{\tilde{\omega}_{0}\left(1-\tilde{y}_{0}\right) \eta}{4 x}
$$

Thus, (24) and (25) become

$$
\begin{gathered}
\int_{x_{0}}^{\infty}\left(E_{1}(s)-\frac{2^{r_{1}}}{\left(r_{1}+1\right)^{r_{1}+1}} \frac{z(s)\left(\alpha^{\prime}(s)\right)^{r_{1}+1}}{\mu_{1}^{r_{1}} s^{2 r_{1}} \alpha^{r_{1}}(s)}\right) \mathrm{d} s \\
=\int_{x_{0}}^{\infty}\left(\frac{\tilde{\omega}_{0}\left(1-\tilde{y}_{0}\right) \eta^{3}}{s}-\frac{2}{\mu_{1} s}\right) \mathrm{d} s
\end{gathered}
$$

and

$$
\begin{aligned}
& \int_{x_{0}}^{\infty}\left(\Phi(s)-\frac{\left(h^{\prime}(s)\right)^{2}}{4 h(s)}\right) \mathrm{d} s \\
& \quad=\int_{x_{0}}^{\infty}\left(\frac{\tilde{\omega}_{0}\left(1-\tilde{y}_{0}\right) \eta}{4 s}-\frac{1}{4 s}\right) \mathrm{d} s .
\end{aligned}
$$

So,

$$
\tilde{\omega}_{0}>\frac{2}{\left(1-\tilde{y}_{0}\right) \eta^{3}}
$$

and

$$
\tilde{\omega}_{0}>\frac{1}{\left(1-\tilde{y}_{0}\right) \eta} \text {. }
$$

From Corollary 2.8, equation (26) is oscillatory if (30) holds. 


\section{Conclusion}

In this work, we proved some new oscillation theorems for (1). New oscillation results are established that complement related contributions to the subject. We used the Riccati technique and the integral averages technique to get some new results to oscillation of equation (1) under the condition $\int_{x_{0}}^{\infty} \frac{1}{z^{1 / r_{1}(s)}} \mathrm{d} s=\infty$. We may say that, in future work, we will study this type of equation under the condition

$$
\int_{x_{0}}^{\infty} \frac{1}{z^{1 / r_{1}}(s)} \mathrm{d} s<\infty
$$

Also we will try to introduce some important oscillation criteria of differential equations of fourth-order and under

$$
\delta(x)=\beta(x)+\tilde{y}(x) \sum_{i=1}^{j} \beta_{i}(\tilde{\theta}(x)) .
$$

\section{Acknowledgements}

The authors thank the editors and the reviewers for their useful comments.

Funding

This research received no external funding.

Availability of data and materials

Not applicable.

Competing interests

The authors declare that they have no competing interests.

Authors' contributions

The authors declare that they have read and approved the final manuscript.

\section{Authors' information}

Not applicable.

\section{Author details}

'Department of Mathematics and Statistics, College of Science, Taif University, P.O. Box 11099, Taif, 21944, Saudi Arabia.

${ }^{2}$ Department of Mathematics, College of Science and Human Studies, Hotat Sudair, Majmaah University, Majmaah, 11952, Saudi Arabia. ${ }^{3}$ Mathematics Departments, College of Science and Arts in Gurayat, Jouf University, Sakaka, 77455, Saudi Arabia. ${ }^{4}$ Department of Mathematics, Faculty of Science, Hadhramout University, Hadhramout, 50512, Yemen. ${ }^{5}$ Department of Electrical and Electronic Engineering Educators, School of Pedagogical and Technological Education (ASPETE), Marousi, 15122, Athens, Greece.

\section{Publisher's Note}

Springer Nature remains neutral with regard to jurisdictional claims in published maps and institutional affiliations.

Received: 16 June 2021 Accepted: 17 August 2021 Published online: 30 August 2021

\section{References}

1. Nofal, T.A., Bazighifan, O., Khedher, K.M., Postolache, M.: More Effective conditions for oscillatory properties of differential equations. Symmetry 13, 278 (2021). https://doi.org/10.3390/sym13020278

2. Althobati, S., Bazighifan, O., Yavuz, M.: Some important criteria for oscillation of non-linear differential equations with middle term. Mathematics 9, 346 (2021)

3. Hale, J.K.: Theory of Functional Differential Equations. Springer, New York (1977)

4. Agarwal, R.P., Zhang, C., Li, T.: Some remarks on oscillation of second order neutral differential equations. Appl. Math. Comput. 274, 178-181 (2016)

5. Bohner, M., Hassan, T.S., Li, T.: Fite-Hille-Wintner-type oscillation criteria for second-order half-linear dynamic equations with deviating arguments. Indag. Math. 29(2), 548-560 (2018)

6. Bohner, M., Li, T.: Kamenev-type criteria for nonlinear damped dynamic equations. Sci. China Math. 58(7), 1445-1452 (2015)

7. Zhang, C., Agarwal, R.P., Bohner, M., Li, T.: Oscillation of fourth-order delay dynamic equations. Sci. China Math. 58(1), 143-160 (2015) 
8. Kumar, M.S., Bazighifan, O., Al-Shaqsi, K., Wannalookkhee, F., Nonlaopon, K.: Symmetry and its role in oscillation of solutions of third-order differential equations. Symmetry 13, 1485 (2021)

9. Erbe, L.H., Kong, Q., Zhang, B.G.: Oscillation Theory for Functional Differential Equations. Marcel Dekker Inc., New York (1995)

10. Chiu, K.-S., Li, T.: Oscillatory and periodic solutions of differential equations with piecewise constant generalized mixed arguments. Math. Nachr. 292, 2153-2164 (2019)

11. Li, T., Rogovchenko, Y.V.: Oscillation criteria for even-order neutral differential equations. Appl. Math. Lett. 61,35-41 (2016)

12. Li, T., Zhang, C., Thandapani, E.: Asymptotic behavior of fourth-order neutral dynamic equations with noncanonical operators. Taiwan. J. Math. 18(4), 1003-1019 (2014)

13. Bazighifan, O., Mofarreh, F., Nonlaopon, K.: On the qualitative behavior of third-order differential equations with a neutral term. Symmetry 13, 1287 (2021)

14. AlGhamdi, A., Bazighifan, O., El-Nabulsi, R.A.: Important criteria for asymptotic properties of nonlinear differentia equations. Mathematics 9, 1659 (2021)

15. Kumar, M.S., Elayaraja, R., Ganesan, V., Bazighifan, O., Al-Shaqsi, K., Nonlaopon, K.: Qualitative behavior of unbounded solutions of neutral differential equations of third-order. Fractal Fract. 5, 95 (2021)

16. Agarwal, R.P., Bohner, M., Li, T., Zhang, C.: A new approach in the study of oscillatory behavior of even-order neutral delay differential equations. Appl. Math. Comput. 225, 787-794 (2013)

17. Agarwal, R.P., Bazighifan, O., Ragusa, M.A.: Nonlinear neutral delay differential equations of fourth-order: oscillation of solutions. Entropy 23, 129 (2021)

18. Bazighifan, O., Alotaibi, H., Mousa, A.A.A.: Neutral delay differential equations: oscillation conditions for the solutions. Symmetry 13, $101(2021)$

19. Chatzarakis, G.E., Elabbasy, E.M., Bazighifan, O.: An oscillation criterion in 4th-order neutral differential equations with a continuously distributed delay. Adv. Differ. Equ. 336, 1-9 (2019)

20. Moaaz, O., Awrejcewicz, J., Bazighifan, O.: A new approach in the study of oscillation criteria of even-order neutral differential equations. Mathematics 12, 1-10 (2020)

21. Li, T., Pintus, N., Viglialoro, G.: Properties of solutions to porous medium problems with different sources and boundary conditions. Z. Angew. Math. Phys. 70(3), Article ID 86 (2019)

22. Frassu, S., Viglialoro, G.: Boundedness for a fully parabolic Keller-Segel model with sublinear segregation and superlinear aggregation. Acta Appl. Math. 171(1), 19 (2021)

23. Li, T., Viglialoro, G.: Boundedness for a nonlocal reaction chemotaxis model even in the attraction-dominated regime. Differ. Integral Equ. 34(5), 315-336 (2021)

24. Bohner, M., Li, T.: Oscillation of second-order p-Laplace dynamic equations with a nonpositive neutral coefficient. Appl. Math. Lett. 37, 72-76 (2014)

25. Li, T., Rogovchenko, Y.V.: On asymptotic behavior of solutions to higher-order sublinear Emden-Fowler delay differential equations. Appl. Math. Lett. 67, 53-59 (2017)

26. Zhang, C., Li, T., Sun, B., Thandapani, E.: On the oscillation of higher-order half-linear delay differential equations. Appl. Math. Lett. 24(9), 1618-1621 (2011)

27. Elabbasy, E.M., Cesarano, C., Bazighifan, O., Moaaz, O.: Asymptotic and oscillatory behavior of solutions of a class of higher order differential equation. Symmetry 11, 1434 (2019)

28. Zhang, C., Agarwal, R.P., Bohner, M., Li, T.: New results for oscillatory behavior of even-order half-linear delay differential equations. Appl. Math. Lett. 26(2), 179-183 (2013)

29. Li, T., Rogovchenko, Y.V.: Oscillation of second-order neutral differential equations. Math. Nachr. 288(10), 1150-1162 (2015)

30. Li, T., Rogovchenko, Y.V.: On the asymptotic behavior of solutions to a class of third-order nonlinear neutral differential equations. Appl. Math. Lett. 105, Article ID 1062 (2020)

31. Dzurina, J., Grace, S.R., Jadlovska, I., Li, T.: Oscillation criteria for second-order Emden-Fowler delay differential equations with a sublinear neutral term. Math. Nachr. 293, 910-922 (2020)

32. Baculikova, B., Dzurina, J., Li, T.: Oscillation results for even-order quasi linear neutral functional differential equations. Electron. J. Differ. Equ. 2011, 143 (2011)

33. Xing, G., Li, T., Zhang, C.: Oscillation of higher-order quasi linear neutral differential equations. Adv. Differ. Equ. 2011, 45 (2011)

34. Chatzarakis, G.E., Grace, S.R., Jadlovská, I., Li, T., Tunç, E: Oscillation criteria for third-order Emden-Fowler differential equations with unbounded neutral coefficients. Complexity 2019, Article ID 5691758 (2019)

35. Li, T., Rogovchenko, Y.V.: Oscillation criteria for second-order superlinear Emden-Fowler neutral differential equations. Monatshefte Math. 184(3), 489-500 (2017)

36. Zhang, C., Li, T., Saker, S.: Oscillation of fourth-order delay differential equations. J. Math. Sci. 201, 296-308 (2014)

37. Kiguradze, I.T., Chanturiya, T.A.: Asymptotic Properties of Solutions of Nonautonomous Ordinary Differential Equations. Kluwer Academic, Dordrecht (1993)

38. Philos, C.G.: A new criterion for the oscillatory and asymptotic behavior of delay differential equations. Bull. Acad. Pol. Sci., Sér. Sci. Math. 39, 61-64 (1981) 